\title{
Utilizzo del sistema Uro-Quick per l'identificazione rapida di batteri produttori di ß-lattamasi a spettro esteso (ESBL)
}

\section{Simone Cagnacci, Fabrizio Cavallini, Elisabetta Maioli, Simona Roveta, Clara Cassanelli, Anna Marchese, Eugenio A. Debbia}

Sezione di Microbiologia - DISCAT, Università di Genova.

Key words: Uro-Quick, ESBL, identificazione.

Objectives: To evaluate the Uro-Quick system for detection of extended-spectrum beta-lactamase (ESBL) among nosocomial strains isolated from urine during a 2 months period.

Methods: A total of 221 strains collected from nosocomial patients were tested for antibiotic susceptibility by the Uro-Quick system. About $10^{6} \mathrm{cell} / \mathrm{ml}$ were used to seed $2.5 \mathrm{ml}$ of broth in vials containing ceftazidime (I0 $\mu \mathrm{g} / \mathrm{mL})$ and ceftazidime $(10 \mu \mathrm{g} / \mathrm{mL})$ plus clavulanic acid $(10 \mu \mathrm{g} / \mathrm{mL})$. After incubation the results were plotted as growth curves. All results were confirmed by Kirby-Bauer method. Control strains were included.

Results: Using an inoculum of $10^{6} \mathrm{cell} / \mathrm{ml}$ the instrument was capable to print out the result after 5 hours of incubation.Among the pathogens studied, 46 strains resulted resistant to ceftazidime (presence of growth). These results were then compared with those obtained with the Kirby-Bauer method, and it was found that among these 46 ceftazidime-resistant clones all were ESBL-producers, even if 12 showed an intermediate, and 12 a susceptible antibiotic phenotype by Kirby-Bauer method.

Conclusion: Present findings suggest that Uro-Quick represents a useful technology to detect ESBL-producing strains especially those that required further confirmatory tests for their identification. The period of time needed to achieve the results, in some cases less than 5 hours, might be an advantage over the usual methodologies.

\section{INTRODUZIONE}

I batteri Gram-negativi resistenti agli antibiotici ß-lattamici in virtù della sintesi di potenti enzimi idrolizzanti detti $\beta$-lattamasi, rappresentano un fenomeno di grosso impatto nella sanità pubblica. La prima $\beta$-lattamasi è stata scoperta negli anni sessanta (3) e denominata TEM-1. Questa proteina, responsabile del $90 \%$ dei casi di resistenza all'ampicilina da parte di Escherichia coli è in grado di idrolizzare le penicilline e le cefalosporine di prima generazione come la cefalotina e la cefaloridina. Essendo questo catalizzatore veicolato da plasmidi ha avuto una rapida diffusione e ha interessato non solo E. coli ma anche altri membri appartenenti alla famiglia delle Enterobacteriaceae. Un'altra ß-lattamasi ritrovata con frequenza piuttosto elevata è la SHV-1, proteina solitamente codificata dal cromosoma in Klebsiella pneumoniae e plasmidica in E. coli.

Negli ultimi anni sono stati messi in commercio un numero elevato di farmaci in grado di contrastare l'azione idrolitica di questi enzimi, una classe di questi antibiotici è rappresentata dalle cefalosporine di terza generazione, denominate anche a spettro esteso, per il fatto che la loro attività in vitro include non solo molti ceppi Gram-negativi ma si estende anche a Pseudomonas. aeruginosa o altri ceppi a rapida crescita non inibiti dalle cefalosporine già in uso da tempo. I microrganismi in grado di sviluppare resistenza nei confron- ti di questi antibiotici sono stati classificati come produttori di B-lattamasi a spettro esteso (ESBL) (1). Attualmente sono state descritte più di 150 diversi tipi di ESBL anche se TEM e SHV rappresentano le classi di enzimi più frequentemente ritrovate.

La maggior parte delle ESBL sono veicolate da plasmidi che ne possono facilitare la diffusione da una specie all'altra. Poiché spesso nei plasmidi troviamo geni che conferiscono resistenze ad altri antibiotici, soprattutto ad aminoglicosidi, cotrimossazolo e tetracicline, si riscontra sovente una concomitante resistenza verso tali molecole (5). Gli stipiti ESBL sono un grave problema emergente per tutti i laboratori di Microbiologia Clinica a livello mondiale. Infatti l'identificazione di questi ceppi richiede test specifici spesso non eseguiti di routine. Il National Commitee for Clinical Laboratory Standards (NCCLS) suggerisce l'esecuzione di indagini di laboratorio per identificare ceppi produttori di ESBL in K. pneumoniae, $K$. oxytoca ed E. coli che manifestano una resistenza a cefpodoxime, ceftazidime, aztreonam, cefotaxime, e ceftriaxone. Per Proteus mirabilis lo screening di routine è sconsigliato, tuttavia per stipiti clinicamente rilevanti, che manifestano una ridotta sensibilità a ceftazidime, cefotaxime o cefpodoxime, e quindi sospetti produttori di ESBL, è consigliato il test di conferma che impiega dischetti di ceftazidime con l'aggiun- 
ta di acido clavulanico (6).

In questo studio preliminare è stato valutato se un sistema automatico Uro-quick utilizzato da tempo per la determinazione della batteriuria, può fornire in tempi più rapidi rispetto alla metodica tradizionale, un'indicazione sulla presenza di ESBL in ceppi isolati da pazienti nosocomiali.

\section{MATERIALI E METODI \\ Ceppi utilizzati}

Per questo studio sono stati utilizzati ceppi Gramnegativi patogeni isolati consecutivamente per 2 mesi da pazienti nosocomiali affetti da infezione alle vie urinarie (tabella 1). 221 ceppi scelti in maniera casuale sono stati analizzati per verificare l'eventuale presenza di produttori di ESBL. L'analisi è stata compiuta utilizzando la metodica prevista dall'NCCLS, il metodo del doppio dischetto e il sistema Uro-Quick.

E. coli ATCC 25922, E. coli ATCC 35218, per i saggi includenti un $\beta$-lattamico e un inibitore di $\beta$ lattamasi, E. coli produttore di ß-lattamasi TEM-2, di ESBL TEM-3 e TEM-4 (2), P. aeruginosa ATCC 27853 e $S$. aureus ATCC 25923 sono stati aggiunti nei saggi di sensibilità come controllo di qualità.

\section{Identificazione di ceppi produttori di ESBL}

La metodica per l'identificazione di stipiti produttori di ESBL suggerita dall'NCCLS prevede l'utilizzo di dischetti contenenti ceftazidime e acido clavulanico. Un dischetto di ceftazidime $(30 \mu \mathrm{g})$ viene addizionato con $10 \mu \mathrm{L}$ di una soluzione di acido clavulanico a $1000 \mu \mathrm{g} / \mathrm{mL}$ e lasciato asciugare per 30 minuti. Il dischetto così preparato viene poi utilizzato per eseguire l'antibiogramma secondo la metodica standard dell'NCCLS. Dopo $18-24$ ore di incubazione a $37^{\circ} \mathrm{C}$ vengono misurati gli aloni di inibizione. Un alone di inibizione di diametro superiore di almeno $5 \mathrm{~mm}$ prodotto dal dischetto contenente ceftazidime più acido clavulanico rispetto al solo ceftazidime indica la presenza di un ceppo produttore di ESBL.

Tecnica di diffusione mediante doppio disco (4). Su una piastra di MH agar sulla quale è stato seminato il ceppo da saggiare vengono posizionati a una distanza di $30 \mathrm{~mm}$ l'uno dall'altro due dischetti contenenti rispettivamente ceftazidime $(30 \mu \mathrm{g})$ e amoxicillina/acido clavulanico $(20 / 10 \mu \mathrm{g})$.

La piastra viene incubata overnight a $37^{\circ} \mathrm{C}$ e la produzione di ESBL è verificata quando l'alone di inibizione attorno al dischetto contenente ceftazidime si espande verso il dischetto di amoxicillina/acido clavulanico.

\section{Sistema URO-QUICK}

L'Uro-Quick (figura I) introdotto da Alifax (Padova, Italia) è uno strumento automatizzato che permette di determinare la sensibilità agli antibiotici in tempi rapidi sulla base del patogeno studiato. I batteri vengono inoculati ad una concentrazione iniziale di $10^{6} \mathrm{CFU} / \mathrm{mL}$ in vials contenenti $2.5 \mathrm{~mL}$ di terreno di coltura e di antibiotico da saggiare alle opportune concentrazioni. Il sistema impiega un raggio laser per valutare la crescita batterica. Dopo 3-5 ore di incubazione a $37^{\circ} \mathrm{C}$ l'apparecchio può fornire i risultati. La presenza o l'assenza di una curva di crescita indica rispettivamente resistenza o sensibilità all' antibiotico saggiato (figura II).

I ceppi sono stati inoculati in vials contenenti ceftazidime $(10 \mu \mathrm{g} / \mathrm{mL})$ e ceftazidime più acido clavulanico $(10 / 10 \mu \mathrm{g} / \mathrm{mL})$ e dopo l'opportuna incubazione a $37^{\circ} \mathrm{C}$ (mai superiore alle 5 ore) lo strumento ha stampato i risultati. L'obiettivo era valutare se l'Uro-Quick fosse stato in grado di evidenziare i ceppi produttori di ESBL come resistenti alla ceftazidime e sensibili alla ceftazidime più acido clavulanico.

Tabella I. Ceppi utilizzati in questo studio provenienti da pazienti nosocomiali

\begin{tabular}{ccc}
\hline CEPPO & NUMERO CEPPI & PERCENTUALE CEPPI \\
\hline E. coli & 106 & $48 \%$ \\
\hline K. pneumoniae & 28 & $12.6 \%$ \\
\hline P. mirabilis & 32 & $14.5 \%$ \\
\hline P. aeruginosa & 22 & $10 \%$ \\
\hline E. cloacae & 8 & $3.6 \%$ \\
\hline C. freundi & 8 & $3.6 \%$ \\
\hline P. stuartii & 4 & $1.8 \%$ \\
\hline M. morganii & 4 & $1.8 \%$ \\
\hline Altri & 9 & $4.1 \%$ \\
\hline
\end{tabular}

Tabella 2. Ceppi produttori di ESBL con relative percentuali di concordanza tra Uro-Quick e metodica classica

\begin{tabular}{cccccc}
\hline \multicolumn{5}{c}{ N. PRODUTTORI ESBL IDENTIFICATI } & CONCORDANZA URO-QUICK/NCCLS \\
\hline Ceppi & Metodica NCCLS & Doppio dischetto & Uro-Quick & \\
\hline E. coli & 13 & 13 & 13 & $100 \%$ \\
\hline K. pneumoniae & 19 & 14 & 19 & $100 \%$ \\
\hline P. mirabilis & 14 & 8 & 12 & $85,7 \%$ \\
\hline Totale & 46 & 35 & 44 & $95,7 \%$ \\
\hline
\end{tabular}




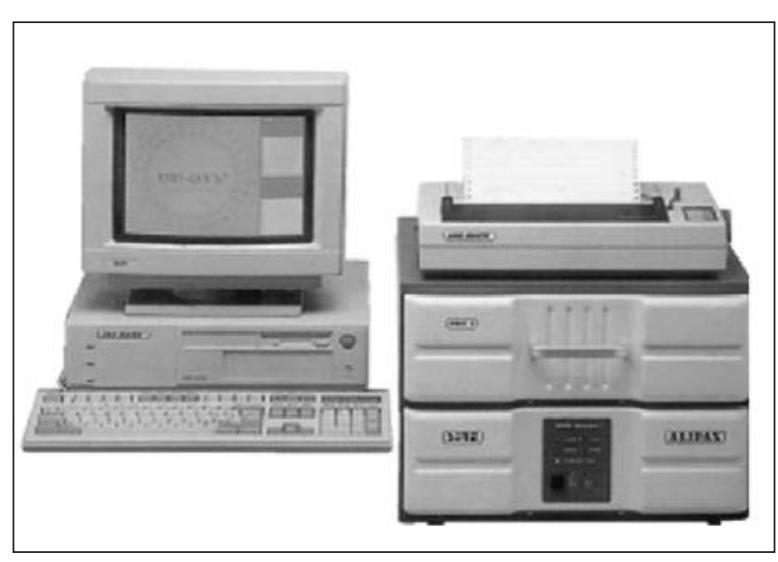

Figura I. Uro-Quick

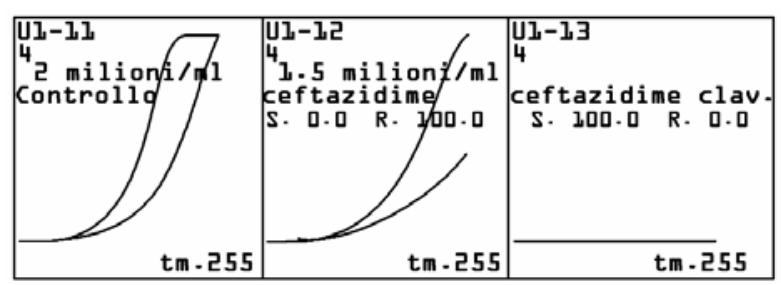

Figura II. Curve di crescita di un ceppo produttore di ESBL

\section{RISULTATI E CONCLUSIONI}

Con la metodica suggerita da NCCLS che prevede l'aggiunta di acido clavulanico al dischetto di ceftazidime, 46 ceppi su un totale di 221 sono risultati produttori di ESBL, di questi 13 E. coli, 19 K. pneumoniae, 14 P. mirabilis.

Con il metodo del doppio dischetto sono stati identificati solamente 35 dei 46 produttori di ESBL. Infatti $5 \mathrm{~K}$. pneumoniae e $6 \mathrm{P}$. mirabilis sono risultati negativi con questo test pur essendo positivi secondo l'NCCLS.

Il sistema Uro-Quick è in grado di seguire la crescita del microrganismo e fornire in tempo reale $\mathrm{i}$ risultati mediante una curva di crescita. Abbiamo quindi inoculato i ceppi nei vials contenenti ceftazidime e ceftazidime più acido clavulanico e messi a incubare nello strumento per 5 ore. Lo scopo era quello di verificare se una differenza tra la curva con il solo antibiotico e quella con l'antibiotico e l'inibitore suicida poteva essere imputabile alla presenza di produttori di ESBL. I risultati ottenuti hanno confermato l' ipotesi infatti in 44 ceppi si è notata una netta differenza tra le due curve e in tutti i casi la metodica classica dell'NCCLS ha confermato la presenza di produttori di ESBL. Di questi 44 ceppi 13 erano E. coli, 19 K. pneumoniae e 12 P. mirabilis.

Dai risultati ottenuti (tabella 2) si evince che la concordanza tra Uro-Quick e metodica classica risulta essere del $100 \%$ in E. coli e K. pneumoniae e del $85.7 \%$ in P. mirabilis. Nel complesso la concordanza tra le due metodiche è stata superiore al $95 \%$.
È stata inoltre effettuata l'analisi con l'antibiogramma Kirby-Bauer utilizzato di routine nei laboratori di analisi. Si deve sottolineare il fatto che con questo metodo dei 46 stipiti produttori di ESBL non tutti hanno mostrato una resistenza evidente al ceftazidime. Infatti 12 hanno manifestato resistenza intermedia e 12 sono risultati sensibili all'antibiotico.

In conclusione, questo studio indica che il sistema Uro-Quick, già utilizzato da tempo nella valutazione della batteriuria, può essere un rapido ed efficace strumento per l'identificazione di batteri produttori di ESBL così da poter effettuare una corretta terapia antibiotica sui pazienti.

\section{RINGRAZIAMENTI}

Si ringrazia la ditta Alifax (Padova) per il sostegno dato durante alcune fasi del lavoro.

\section{BIBLIOGRAFIA}

1. Bradford PA. Exented-spectrum B-lactamases in the $21^{\text {st }}$ century: characterization, epidemiology, and detection of this important resistance threat. Clin Microbiol Rev 2001; 14: 933-51.

2. Casini Lemmi M, Dho G, Manno G, Marchese A, Debbia EA. Identificazione e resistenza agli antibiotici di ceppi ben caratterizzati analizzati in laboratori liguri. Microbiologia Medica 2004; 19: 14-22.

3. Datta N, Kontomichalou P. Penicillinase synthesis controlled by infextions R Factor in enterobacteriacee. Nature, 1965; 208: 239-44.

4. Livermore DM, Brown DFJ. Detection of ß-lactamasesmediated resistance, 2001; 48: suppl. S1, 59-64.

5. Jacoby GA, Sutton L. Properties of plasmids responsible for production of exended-spectrum B-lactamases. Antimicrob Agent Chemother. 1991; 35: 164-9.

6. National Committee for Clinical Laboratory Standards (NCCLS). Performance standards for antimicrobial susceptibility testing, NCCLS 2005.

7. Roveta S, Debbia EA, Marchese A. Valutazione della sensibilità agli antibiotici di uropatogeni mediante sistema Uro-Quick direttamente su campioni di urine e confronto con il metodo Kirby-Bauer. Gimmoc 2003; 7: 67-74.

8. Roveta S, Marchese A, Debbia EA. Evaluation of the Uro-Quick, a new rapid automated system, for the detection of well charterized antibiotic-resistant bacteria. J Chemother 2004; 16: 107-18.

\section{Eugenio A. Debbia}

Università degli Studi di Genova DISCAT -Sezione di Microbiologia Largo Rosanna Benzi 10 - 16132 Genova - Italia Tel. ++010-3537655; Fax ++010-3537698 E-mail: eugenio.debbia@unige.it 